\title{
Robust PID Controller Tuning with Specification on Modulus Margin
}

\author{
D. Garcia, A. Karimi and R. Longchamp
}

\author{
Laboratoire d'Automatique, \\ École Polytechnique Fédérale de Lausanne (EPFL), \\ CH-1015 Lausanne, Switzerland. email: daniel.garciaeepfl.ch
}

\begin{abstract}
An iterative controller tuning method based on a frequency criterion is proposed in this paper. The frequency criterion is defined as the weighted sum of squared errors between the desired and actual value of the modulus margin (inverse of the infinity norm of the sensitivity function), phase margin and crossover frequency. The criterion is minimized using the iterative Gauss-Newton algorithm. The gradient and Hessian of the criterion can be expressed in terms of the derivatives of the open-loop system with respect to the frequency. These derivatives, as well as the robustness margins and the related frequencies are computed using a plant model. Simulation examples illustrate the effectiveness and the simplicity of the proposed method for controller tuning.
\end{abstract}

\section{INTRODUCTION}

Simple controllers, such as the conventional PID controllers, are undeniably the most commonly used control algorithms in industrial processes. In spite of their very simple structure, they often perform well and meet the specifications, provided that their parameters are properly tuned. Consequently, there is an interest in systematic tuning methods for these industrial plant controllers.

Available methods usually lead to controllers achieving specified loop specifications. These consist on robustness margins and related frequencies. Their values are crucial for the stability robustness and they represent also the time domain performances of the closed-loop system.

The classical loop frequency specifications are habitually expressed in terms of phase margin, gain margin and crossover frequency [3], [5], [7]. But gain and phase margins only measure the distance from the critical point to the Nyquist curve in certain specific directions. If one of them is small, the closed-loop system is close to instability. However, for some systems like resonant mechanical processes, this can also be the case if both margins are relatively large.

On the other hand, the maximum value of the sensitivity function constitutes a much better robustness margin. This value is simply the inverse of the shortest distance from the Nyquist curve to the critical point -1 . It quantifies how sensitive is the closed-loop system to variations of the considered plant. It also gives an upper bound for the disturbance amplification by the closed-loop system. Furthermore, it has been pointed out in [4] that the infinity norm of the sensitivity function is a good design parameter in the sense that different processes designed with the same specified value exhibit very similar time responses, in a normalized time scale.
The model-based controller design method proposed in this paper is based on the Gauss-Newton iterative procedure to minimize iteratively a frequency criterion. Different frequency specifications are considered: the inverse of the maximum value of the sensitivity function (called the modulus margin) as well as the phase margin and the crossover frequency. The first two specifications ensure for processes encountered in practice the stability and robustness of the closed-loop system. The crossover frequency, which is related to the rise time of the closed-loop system can also be taken into account to represent the time-domain performance. The frequency criterion is then defined as the weighted sum of squared errors between the desired specifications and the actual values of the frequency characteristics. The gradient and the Hessian of the criterion can be expressed in terms of the derivatives of the amplitude and phase of the openloop frequency response, that can be computed analytically thanks to the plant model. In few iterations the controller minimizing the criterion is obtained. The specified robustness and performances are thus satisfied on the resulting closedloop system.

The paper is organized as follows: The controller design procedure is presented in Section II. The choice of the specification values are suggested is Section III. Simulation examples are provided in Section IV and finally some concluding remarks are offered in Section V.

\section{Controller DESign PRocedure}

The proposed method assumes that a linear process model $G(s)$ is given that can also include a time delay. If no model of the plant is available at the beginning of the design procedure, there exist nowadays many identification techniques to obtain a first-order plus dead-time or a second order plus dead-time model that approximate well the plant in the important frequency region for controller design. Different methods are proposed in [6] through the analysis of the open-loop step response. Alternatively simple models can be derived from relay feedback experiments [5] by measuring one [3] or more [9] points on the frequency response of the process. In [9] an identification procedure is presented to obtain a second-order model for an extensive class of linear processes including low or high order plant with small or large dead time and monotonic or oscillatory responses.

In addition to a plant model, another prior requirement for 
the design procedure is the existence of an initial stabilizing controller. If no controller already exists, the simplest way to obtain one is the use of experimental tuning rules, like the well-known Ziegler-Nichols method. Generally the closed-loop system obtained with such initial controllers is stable with acceptable robustness margins. As a result, the design procedure will converge to the desired values in few iterations.

The frequency criterion to minimize is defined as follows:

$$
\begin{aligned}
J(\rho)=\frac{1}{2}\left(\lambda_{1}\left(M_{a}(\rho)-M_{d}\right)^{2}\right. & +\lambda_{2}\left(\Phi_{a}(\rho)-\Phi_{d}\right)^{2} \\
& \left.+\lambda_{3}\left(\omega_{a}(\rho)-\omega_{d}\right)^{2}\right)
\end{aligned}
$$

where $\rho$ is the vector of the controller parameters of dimension $n_{\rho}, \lambda_{1}, \lambda_{2}$ and $\lambda_{3}$ are weighting factors, $M_{a}$ and $M_{d}$ are respectively the actual and desired inverse of the infinity norm of the sensitivity function, which is also called the modulus margin. $\Phi_{a}$ and $\Phi_{d}$ are the actual and desired phase margin and $\omega_{a}$ and $\omega_{d}$ the actual and desired crossover frequency. It should be noted that the phase margin $\Phi_{a}$, the crossover frequency $\omega_{a}$ and the modulus margin $M_{a}$ can easily be computed numerically using the plant model and the in hand controller transfer function.

Then the controller parameters minimizing the criterion can be obtain iteratively by the Gauss-Newton method:

$$
\rho_{i+1}=\rho_{i}-\gamma_{i} R^{-1} J^{\prime}\left(\rho_{i}\right)
$$

where $i$ is the iteration number, $\gamma_{i}$ the step size, $R$ a positive definite matrix of dimension $n_{\rho} \times n_{\rho}$ that can be chosen equal to the Hessian $H$ for a fast convergence and $J^{\prime}(\rho)$ is the gradient of the criterion with respect to $\rho$. Note that this iterative algorithm gives only a local minimum of the criterion, so the initial choice of controller plays an important role.

The gradient of the criterion is given by:

$$
\begin{aligned}
J^{\prime}(\rho)=\lambda_{1}\left(M_{a}-M_{d}\right) \frac{d M_{a}}{d \rho}+ & \lambda_{2}\left(\Phi_{a}-\Phi_{d}\right) \frac{d \Phi_{a}}{d \rho} \\
& +\lambda_{3}\left(\omega_{a}-\omega_{d}\right) \frac{d \omega_{a}}{d \rho}
\end{aligned}
$$

and the Hessian of the criterion is given by:

$$
\begin{aligned}
H(\rho)= & \lambda_{1} \frac{d M_{a}}{d \rho}\left(\frac{d M_{a}}{d \rho}\right)^{T}+\lambda_{2} \frac{d \Phi_{a}}{d \rho}\left(\frac{d \Phi_{a}}{d \rho}\right)^{T} \\
+ & \lambda_{3} \frac{\partial \omega_{a}}{\partial \rho}\left(\frac{\partial \omega_{a}}{\partial \rho}\right)^{T}+\lambda_{1}\left(M_{a}-M_{d}\right) \frac{d^{2} M_{a}}{d \rho^{2}} \\
& +\lambda_{2}\left(\Phi_{a}-\Phi_{d}\right) \frac{d^{2} \Phi_{a}}{d \rho^{2}}+\lambda_{3}\left(\omega_{a}-\omega_{d}\right) \frac{d^{2} \omega_{a}}{d \rho^{2}}
\end{aligned}
$$

The last three terms containing the second derivatives can be neglected because they are small especially in the neighborhood of the optimum. In addition this simplifies largely the computation of the Hessian, which can be computed without any additional information, since the remaining terms are also present in the gradient. Hence:

$$
\begin{aligned}
H \approx \lambda_{1} \frac{d M_{a}}{d \rho}\left(\frac{d M_{a}}{d \rho}\right)^{T}+\lambda_{2} \frac{d \Phi_{a}}{d \rho} & \left(\frac{d \Phi_{a}}{d \rho}\right)^{T} \\
& +\lambda_{3} \frac{d \omega_{a}}{d \rho}\left(\frac{d \omega_{a}}{d \rho}\right)^{T}
\end{aligned}
$$

It now remains to determine the derivatives of $M_{a}, \Phi_{a}$ and $\omega_{a}$ with respect to the controller parameters $\rho$.

\section{A. Derivative of the Modulus Margin}

Consider $\omega_{M}$ as the frequency where the sensitivity function reaches its maximum value. $\omega_{M}$, which is thus the frequency of the open-loop system at the modulus margin, is obviously a function of the controller parameters $\rho$. The term $M_{a}^{\prime}\left(=\frac{d M_{a}}{d \rho}\right)$ can then be computed through the chain rule as follows:

$$
M_{a}^{\prime}=\frac{\partial M_{a}}{\partial \rho}+\left.\frac{\partial M_{a}}{\partial \omega}\right|_{\omega_{M}} \frac{\partial \omega_{M}}{\partial \rho}
$$

Since the modulus margin is the shortest distance between the Nyquist curve of the open-loop system and the critical point -1 , the first term in the above equation can be written as:

$$
\frac{\partial M_{a}}{\partial \rho}=\frac{\partial\left|1+K\left(j \omega_{M}\right) G\left(j \omega_{M}\right)\right|}{\partial \rho}
$$

where $K(s)$ represents the transfer function of the controller. The numerical value of Eq. (7) can easily be computed analytically and does not contain any derivatives of the plant model, since these derivatives with respect to the controller parameters are zero.

The second term:

$$
\left.\frac{\partial M_{a}}{\partial \omega}\right|_{\omega_{M}}=\left.\frac{\partial|1+K(j \omega) G(j \omega)|}{\partial \omega}\right|_{\omega_{M}}
$$

is the derivative of $|1+K(j \omega) G(j \omega)|$ with respect to the frequency at the minimum value of this function. Since the function $|1+K(j \omega) G(j \omega)|$ and its first derivative with respect to the frequency are continuous functions, the latter is obviously zero at the minimum value of the function. Thus one has:

$$
\left.\frac{\partial M_{a}}{\partial \omega}\right|_{\omega_{M}}=0
$$

and consequently:

$$
M_{a}^{\prime}=\frac{\partial\left|1+K\left(j \omega_{M}\right) G\left(j \omega_{M}\right)\right|}{\partial \rho}
$$

The derivative of the modulus margin with respect to the controller parameters can thus be computed analytically without any derivatives of the plant model. Since the controller structure and parameters are known, the only knowledge of $G\left(j \omega_{M}\right)$ is sufficient for determining $M_{a}^{\prime}$. This information could be used for a data-driven controller tuning procedure in future works. By measuring only the modulus margin and the 
corresponding frequency with an experiment [2], the exact value of the derivatives $M_{a}^{\prime}$ is directly known.

\section{B. Derivative of the Crossover Frequency}

To compute the derivative of the crossover frequency, we use the fact that the loop gain at $\omega_{a}$ is by definition always equal to 1 . Its derivative with respect to $\rho$ is therefore zero [7]:

$$
\frac{d\left|L\left(j \omega_{a}\right)\right|}{d \rho}=\frac{\partial\left|L\left(j \omega_{a}\right)\right|}{\partial \rho}+\left.\frac{\partial|L(j \omega)|}{\partial \omega}\right|_{\omega_{a}} \frac{\partial \omega_{a}}{\partial \rho}=0
$$

where $L(j \omega)=K(j \omega) G(j \omega)$. The first term in the above equation is equal to $\partial\left|K\left(j \omega_{a}\right)\right| / \partial \rho$. Thus $d \omega_{a} / d \rho$ can be written as follows:

$$
\frac{d \omega_{a}}{d \rho}=\frac{\partial \omega_{a}}{\partial \rho}=-\frac{\partial\left|K\left(j \omega_{a}\right)\right|}{\partial \rho}\left(\left.\frac{\partial|L(j \omega)|}{\partial \omega}\right|_{\omega_{a}}\right)^{-1}
$$

and can easily be computed at each iteration.

\section{Derivative of the Phase Margin}

The derivative of the phase margin with respect to the controller parameters can be computed through the chain rule as follows [7]:

$$
\Phi_{a}^{\prime}=\frac{d \Phi_{a}}{d \rho}=\frac{\partial \Phi_{a}}{\partial \rho}+\left.\frac{\partial \Phi_{a}}{\partial \omega}\right|_{\omega_{a}} \frac{\partial \omega_{a}}{\partial \rho}
$$

Now replacing $\Phi_{a}$ in the above equation by $\angle L\left(j \omega_{a}\right)+\pi$ gives:

$$
\Phi_{a}^{\prime}=\frac{\partial \angle L\left(j \omega_{a}\right)}{\partial \rho}+\left.\frac{\partial \angle L(j \omega)}{\partial \omega}\right|_{\omega_{a}} \frac{\partial \omega_{a}}{\partial \rho}
$$

The first term in Eq. (14) is equal to $\partial \angle K\left(j \omega_{a}\right) / \partial \rho$. The first two terms can be computed analytically using the model of the plant and the controller and the last term has been determined in Eq. (12).

\section{Choice of SPECIFICATIONS}

A phase margin between $30^{\circ}$ and $60^{\circ}$ is usually adopted in practice. Recommended value for the largest magnitude of the sensitivity function is typically between 1.3 and 2 which gives a modulus margin between 0.3 and 0.5 . The crossover frequency however should be chosen taking into account the plant dynamics. Guiding rules on the choice of this specification can however be formulated. If the desired rise-time $\tau$ for the closed-loop system is approximatively known, the rule:

$$
\omega_{d} \approx \frac{2.3}{\tau}
$$

gives often satisfactory results. It comes from the consideration that the closed-loop system behaves roughly like a second-order system. It applies approximately and only to stable minimum-phase system. On the other hand, the crossover frequency is usually near to the closed-loop bandwidth, which is often a given specification.
If the rise time or the bandwidth of the closed-loop system are not specified, it is not necessary to give a specification on the crossover frequency. The corresponding terms can be simply removed from the criterion of Eq. (1). Then a controller having only two parameters (PI or PD controller) can be sufficient to minimize the criterion. In the case of a PID controller, the number $n_{\rho}$ of the controller parameters can be set to two by choosing a constant ratio between the integral and derivative time. In [6] it is pointed out that the ratio given by the Ziegler-Nichols tuning rules $T_{i}=4 T_{d}$ is appropriate for many typical industrial processes. Another choice is $T_{i}=2.5 T_{d}$ which also gives good results for a certain number of plant models [8].

Concerning the weighting factors, it has been shown in [1], that their values do not have any influence on the results (in so far they are chosen different from zero), since the dimension $n_{\rho}$ of the controller parameter vector is equal to the number of specifications. In this case, it is recommended to choose each weighting factor equal to the squared inverse of the corresponding specification, in order to normalize the terms in the criterion.

\section{Simulation EXAMPLES}

Now four different plant models are considered to illustrate the proposed method:

$$
\begin{aligned}
G_{p 1}(s) & =\frac{1}{(s+1)^{2}} e^{-0.2 s} \\
G_{p 2}(s) & =\frac{1}{(s+1)^{2}(s+4)} \\
G_{p 3}(s) & =\frac{-0.2 s+1}{(s+1)^{2}} e^{-0.1 s} \\
G_{p 4}(s) & =\frac{1}{s^{2}+1.5 s+1} e^{-0.1 s}
\end{aligned}
$$

Since it is usual to include a noise filter for the derivative term in the PID controller, the following controller structure is used in this section:

$$
K(s)=K p\left(1+\frac{1}{T_{i} s}+\frac{T_{d} s}{\frac{T_{d}}{20} s+1}\right)
$$

For each plant model an initial PID controller is first designed using the Ziegler-Nichols tuning rules. The proposed method is then used to adjust the robustness margins and thus to improve the stability and performances of the closed-loop system. The specifications are set to $60^{\circ}$ for the phase margin and 1.40 for the infinity norm of the sensitivity function. No specifications are however given for the crossover frequency and the constant ratio $T_{i}=4 T_{d}$ is chosen between the integral and derivative time. The resulting controllers are then compared with those obtained with the Kappa-Tau method [6], which is an empirical method that also uses the modulus margin as a design parameter. The specified value for the maximum of the sensitivity (the inverse of the modulus margin) is the same for both methods. 
TABLE I

SIMULATION RESULTS

\begin{tabular}{llcccccccc}
\hline Process & Method & $K_{p}$ & $T_{i}$ & $T_{d}$ & $M_{s}$ & $\Phi_{a}$ & $\omega_{a}$ & $o$ & $t_{s}$ \\
\hline \multirow{2}{*}{$G_{p 1}$} & $\kappa-\tau$ & 3.39 & 1.31 & 0.322 & 1.45 & 48.3 & 1.56 & 20.2 & 5.06 \\
& Proposed & 3.57 & 1.64 & 0.41 & 1.40 & 60.0 & 1.69 & 8.06 & 3.45 \\
$G_{p 2}$ & $\kappa-\tau$ & 16.0 & 1.40 & 0.338 & 1.48 & 46.8 & 1.66 & 22.9 & 4.65 \\
& Proposed & 19.9 & 2.10 & 0.526 & 1.40 & 59.9 & 2.33 & 7.60 & 2.74 \\
\multirow{2}{*}{$G_{p 3}$} & $\kappa-\tau$ & 2.11 & 1.45 & 0.367 & 1.43 & 53.2 & 1.11 & 13.3 & 6.08 \\
& Proposed & 2.15 & 1.64 & 0.41 & 1.40 & 59.9 & 1.11 & 6.82 & 4.67 \\
\multirow{2}{*}{$G_{p 4}$} & $\kappa-\tau$ & 4.96 & 1.10 & 0.263 & 1.65 & 37.38 & 2.21 & 32.17 & 6.07 \\
& Proposed & 5.76 & 1.88 & 0.470 & 1.40 & 59.8 & 3.08 & 7.08 & 4.02 \\
\hline
\end{tabular}

To facilitate the comparison between the controllers, the results are given in tables and graphs. Step responses obtained in closed-loop with controllers tuned using our method and Kappa-Tau rules are compared in Fig. 1 for all plant models. The details of design, the related performances and robustness achievements are shown in Table I, where $M_{s}$ represents the inverse of the achieved modulus margin, $O$ stands for the overshoot in percent and $t_{s}$ for the settling time to $1 \%([s])$. These results show that the proposed controllers improve considerably the time-domain performances of the closed-loop system. In each case, both overshoot and settling time have been clearly reduced. Furthermore there is a large similarity between the responses obtained with the same values of the specifications. This shows that specifications on phase and on the modulus margins constitute suitable design parameters.

Fig. 2 shows the Nyquist plots of the loop transfer functions for each simulation model with the controller resulting from the Kappa-Tau rules and the proposed one. A circle with the radius corresponding to the specified modulus margin (minimum distance from the Nyquist curve to the critical point -1 ) is also shown in the figure. The Nyquist curves of the open-loop systems designed with the proposed method fill the requirement contrary to those designed with the KappaTau tuning rules. Here also it can be observed that by controlling both the phase and the modulus margins, the Nyquist diagram of open-loop transfer functions are very similar in a large frequency band for different plants. Furthermore it can also be seen that the modulus margin constitutes a much better robustness and performance indicator than the classical gain margin. The latter does not capture the essence of a control problem. In other words the choice of an appropriate value for the gain margin is not a priori known and depends especially on the system structure and parameters. For a first or a second order system with negligible time delay, the gain margin should be chosen very large $\left(G_{p 2}(s)\right)$ contrary to that of a higher order system or a system with a large time delay $\left(G_{p 1}(s)\right)$. In addition, a maximum value of the sensitivity function $M_{s}$ ensures a lower bound for the gain margin of
$\frac{M_{s}}{M_{s}-1}$. In the proposed simulation examples the maximum value of the sensitivity function $M_{s}=1.40$ guaranties a gain margin larger than 3.5.

\section{CONCLUSiON}

An iterative method for tuning the controller parameters with specifications on the modulus margin, phase margin and crossover frequency has been proposed. A frequency criterion, defined as the weighted sum of squared errors between the desired and actual value of the specifications has been minimized iteratively using the Gauss-Newton algorithm. A plant model has been used to estimate the actual robustness margins and the related frequencies as well as the derivatives of amplitude and phase of the plant at the crossover frequency with respect to the frequency. Simulation results show that the tuning method converges effectively to the minimum of the criterion and can thus be used for the auto-tuning of industrial plants. The proposed method can be extended to a data-driven controller tuning procedure without any parametric model of the plant. Finally, In further works the robustness against the model uncertainty will be investigated by including a weighting filter in the criterion.

\section{REFERENCES}

[1] D. Garcia, A. Karimi, and R. Longchamp. Data-driven controller tuning based on a frequency criterion. In CDC03, December 2003.

[2] D. Garcia, A. Karimi, and R. Longchamp. Infinity norm measurement of sensitivity function based on limit cycles in a closed-loop experiment. In ECC03, September 2003.

[3] W. K. Ho, C. C. Hang, and L. S. Cao. Tuning of PID controllers based on gain and phase margin specifications. Automatica, 31(3):497-502, 1995.

[4] K. J. Aström, H. Panagopoulos, and T. Hägglund. Design of PI controllers based on non-convex optimisation. Automatica, 34(5):585-601, 1998.

[5] K. J. Aström and T. Hägglund. Automatic tuning of simple regulators with specifications on phase and amplitude margins. Automatica, 20(5):645-651, 1984. 

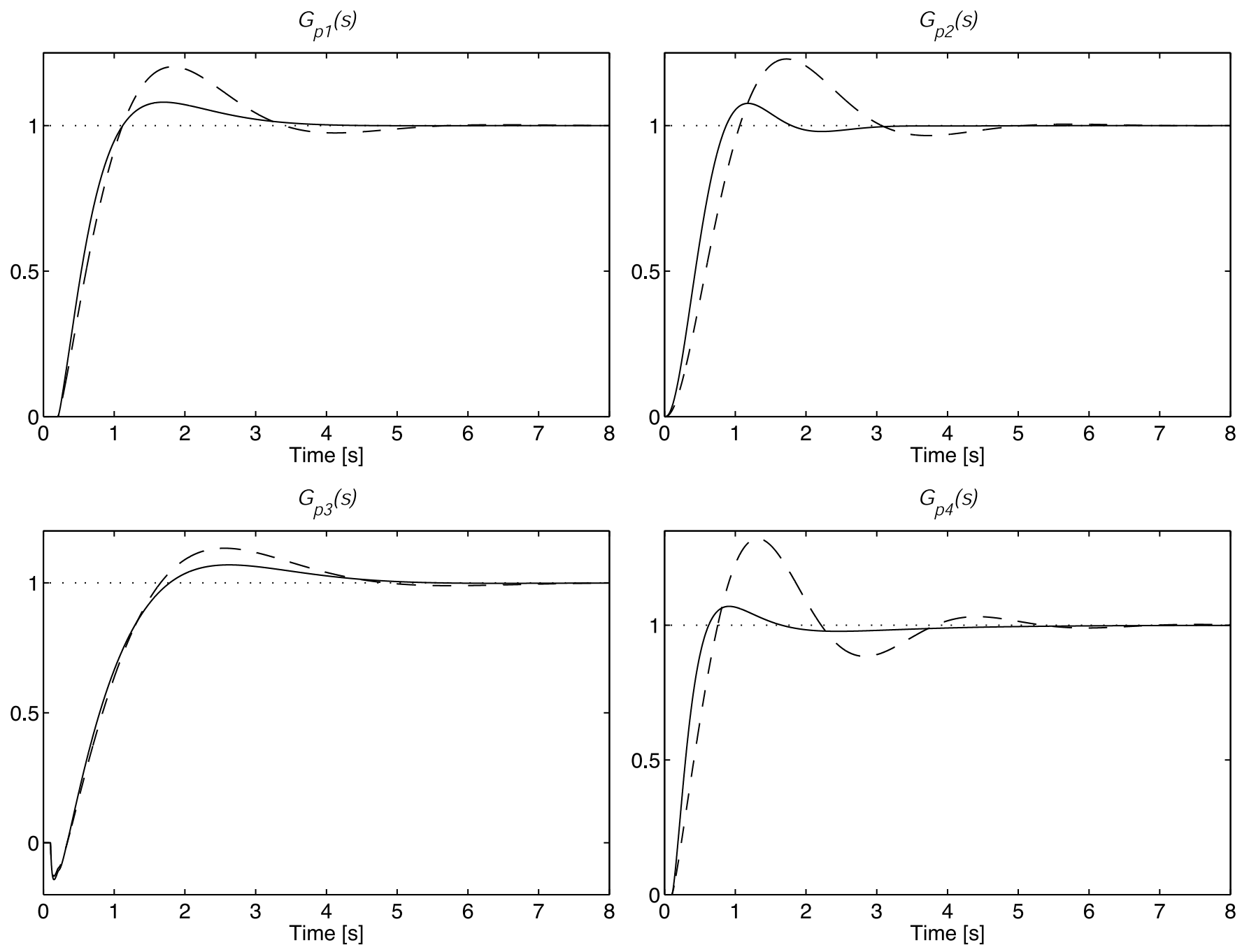

Fig. 1. Step responses (dashed: Kappa-Tau, solid: proposed)

[6] K. J. Aström and T. Hägglund. PID Controllers: Theory, Design and Tuning. Instrument Society of America, 2nd edition, 1995.

[7] A. Karimi, D. Garcia, and R. Longchamp. Iterative controller tuning using Bode's integrals. In $C D C$, FrM076, pages 4227-4232, December 2002.

[8] B. Kristiansson, B. Lennartson, and C. M. Fransson. From PI to $h_{\infty}$ control in a unified framework. In 39th IEEE-CDC, Sydney, Australia, pages 2740-2745, 2000.

[9] Q. G. Wang, T. H. Lee, H. W. Fung, Q. Bi, and Y. Zhang. PID tuning for improved performance. IEEE Transactions on CST, 7(4):3984-3989, 1999. 

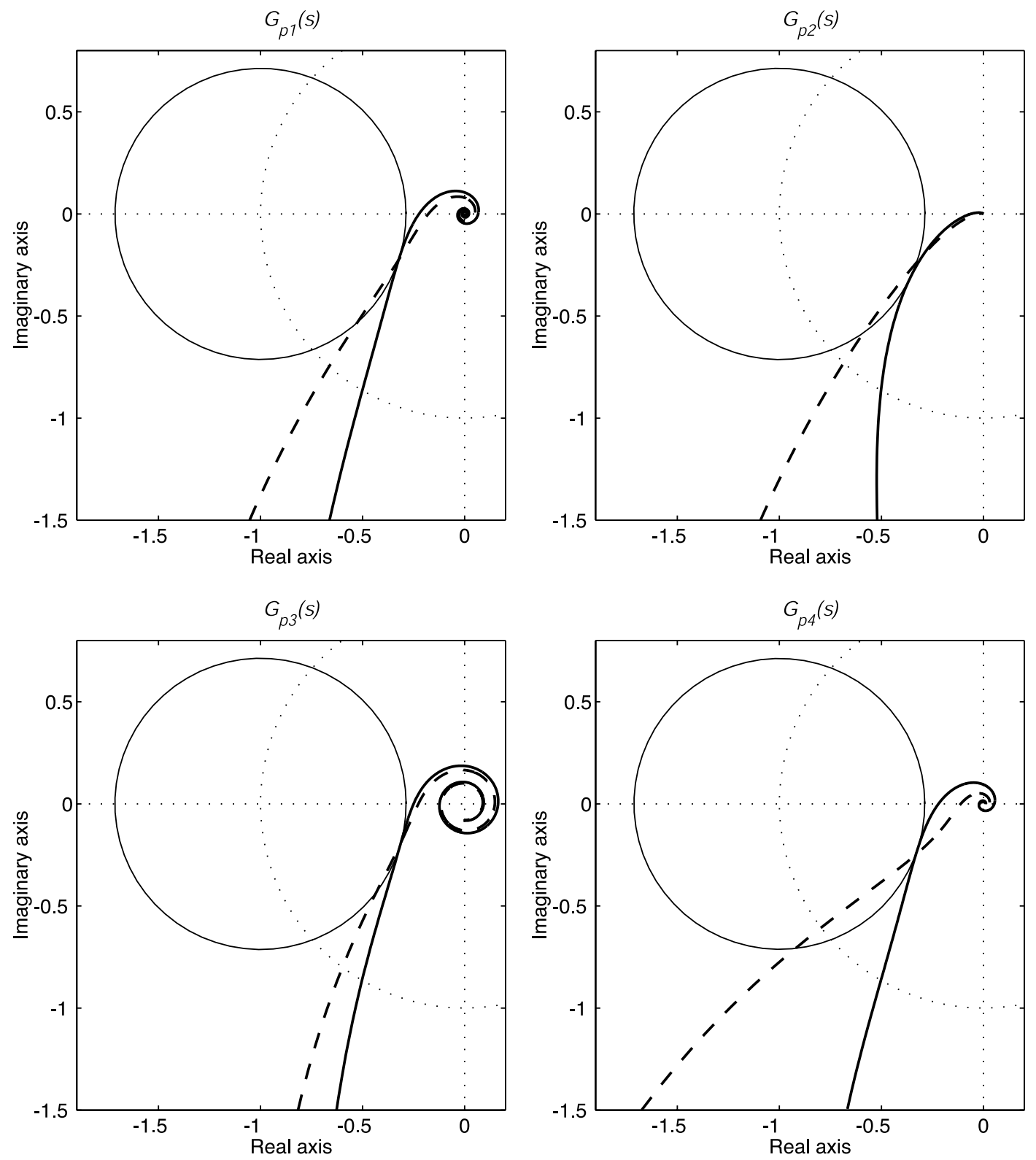

Fig. 2. Nyquist plots (dashed: Kappa-Tau, solid: proposed) 